\title{
Qualitative und quantitative Methoden der Organisationsforschung - ein Überblick
}

\author{
Stefan Kühl, Petra Strodtholz und Andreas Taffertshofer
}

Obwohl die Organisationsforschung weder über eine allgemein akzeptierte Organisationstheorie noch über eine einheitliche methodische Herangehensweise verfügt, sind sich doch die meisten Forscher darin einig, was eine Organisation ist - und was nicht. Genauso wie die meisten Praktiker zu wissen scheinen, ob sie es mit einer Organisation zu tun haben oder nicht, bildet sich auch unter Organisationsforschern bei allen theoretischen und methodischen Gegensätzen meist schnell Konsens, ob sie überhaupt mit einer Organisation befasst sind oder nicht.

In modernen Gesellschaften unterscheiden sich Organisationen von Gesprächen in Kneipen und Zusammenkünften in Fahrstühlen, von Familien und Gruppen, von sozialen Bewegungen und von gesellschaftlichen Teilsystemen wie der Wirtschaft oder der Politik durch drei Merkmale: durch Zwecke, durch Hierarchien und durch Mitgliedschaften. Besonders die systemtheoretische Organisationsforschung hat herausgearbeitet, dass in der Strukturierung moderner Gesellschaften die Bedeutung von Zwecken, Hierarchien und Mitgliedschaften abnimmt, dass diese aber als zentrales Strukturierungsmerkmal von Organisationen einen zunehmend prominenten Platz bekommen (vgl. in Anlehnung an Luhmann hierzu besonders Kieserling 1994; siehe auch Kühl 2000a und Tacke 2001).

Demnach verzichten moderne Gesellschaften im Gegensatz zu den Gesellschaften des Altertums oder des Mittelalters darauf, sich übergeordneten Zwecken wie der Beglückung der Bevölkerung oder der Befolgung göttlicher Gebote zu verschreiben. Ganz anders Organisationen: Egal, ob es sich um eine staatliche Verwaltung, ein Unternehmen in der Internet-Branche, ein Krankenhaus oder eine Gewerkschaft handelt - konkrete Zwecke wie eine mehr oder minder freundliche Befriedigung von Anfragen nach Aufenthaltsgenehmigungen, die Eroberung des Markts mit einem neuen Web-Browser, die kostengünstige und möglichst effektive Behandlung von Patienten oder aber der Abschluss eines Tarifvertrags mit hohen Lohnsteigerungen spielen eine zentrale Rolle in der Ausrichtung von Organisationen (vgl. Luhmann 1973, S. 87 ff.; 1997, S. 826 ff.).

Auch das Management des Eintritts und Austritts - die Bestimmung von Mitgliedschaften - handhaben Organisationen anders als moderne Gesellschaften. Ein totaler Ausschluss aus der Gesellschaft findet nur noch in Ausnahmefällen statt. Auf die Aberkennung der Staatsbürgerschaft verzichten die meisten modernen Staaten (ausgenommen vielleicht die Todesstrafe in einigen ,zivilisierten Ländern“). Das Management der Mitgliedschaft ist dagegen ein zentrales Merkmal von Organisationen. Über die Mitgliedschaft wird trennscharf festgelegt, wer zu einer Einrichtung gehört und wer nicht. Dadurch werden Grenzen geschaffen, in denen sich die Mitglieder (und eben nur die Mitglieder) den Regeln der Organisation zu unterwerfen haben (Luhmann 1995, S. 16).

Schließlich verlieren auch Hierarchien in der Gesellschaft an Bedeutung, während sie für die Strukturierung von Organisationen zentral bleiben. Es gibt in modernen Gesell- 
schaften keine Fürsten, Könige oder Kaiser mehr, die über Befehls- und Anweisungsketten in die verschiedenen Lebensbereiche der Bevölkerung hineinregieren könnten. Eine solche Gesellschaft gälte als diktatorisch, rückständig und unmodern. In der modernen Gesellschaft akzeptiert niemand die Präsidentin als oberste Vorgesetzte einer Befehlshierarchie. Einzige Ausnahme: Mitarbeiter der Präsidialorganisation. Im Gegensatz zu modernen Gesellschaften sind Organisationen zentral über Hierarchien strukturiert. Aller Enthierarchisierungs- und Dezentralisierungsrhetorik zum Trotz können wir uns komplexere Organisationen ohne Hierarchie nicht vorstellen. Erst die Hierarchie stellt sicher, dass die Anweisungen und Zusagen der Spitze auch umgesetzt werden. Sie gewährleistet somit, dass Verbände, Verwaltungen und Unternehmen überhaupt als berechenbare kollektive Akteure auftreten können (Luhmann 1997, S. 834).

\section{Das quantitative Paradigma in der Organisationsforschung}

In der frühen Organisationsforschung wurde die Organisation vom Zweck her gedacht. Organisationszwecke wie etwa die Erzielung eines höheren Marktanteils im Hörbuchbereich oder die Verringerung der Mortalitätsrate im Krankenhaus galten als gesetzte Zwecke, an denen sich die jeweilige Organisation zu orientieren hat. Im Verlauf der Zielverfolgung und -realisation bilden Organisationen, so die Grundüberzeugung, formale Strukturen aus, um die Aktivitäten ihrer Mitglieder sowie den Technik- und Materialeinsatz zielgerecht zu steuern. Das Regelwerk, die Hierarchie und die internen Arbeitsabläufe betrachteten die frühen Vertreter des Fachs lediglich als Mittel, mit dem die Organisation ihre Zwecke und Ziele zu erreichen sucht (vgl. Kieser/Segler 1981).

Dieses „Denken“ der Organisation von ihren Zwecken her prägte maßgeblich auch den Methodeneinsatz der frühen Organisationsforschung. Mit Blick auf die Effektivität und Effizienz einer Organisation interessierte sich die junge Disziplin vor allem für ausgesuchte Zweck-Mittel-Relationen, die sie mit Hilfe standardisierter Fragebögen und statistischer Auswertungsverfahren ausführlich beforschte. Im Mittelpunkt der Untersuchungen standen in der Regel eine überschaubare Anzahl von dem Weber'schen Bürokratiemodell entlehnten Strukturvariablen (z. B. Leitungsspanne, Aufgabenspezialisierung und standardisierung) sowie deren potenzielle Determinanten in der Organisation oder in ihrem Umfeld (vgl. Blau 1955; Hall 1963; Udy 1965; Pugh/Hickson 1976).

Der erkenntnistheoretischen Tradition des logischen Positivismus verpflichtet, gehen die Vertreter der quantitativen Organisationsforschung bis heute ganz selbstverständlich davon aus, dass sie einer objektiven sozialen Realität mit verallgemeinerbaren Gesetzmäßigkeiten gegenüberstehen. Ihr Ziel ist die Entwicklung eines kumulativen Wissensbestandes durch die systematische Überprüfung und mathematische Darstellung bereits vorgefasster Kausalitätsannahmen. Ihr Ehrgeiz liegt darin, mittels hochgradig standardisierter Instrumente und Forschungsstrategien möglichst wertfreie Erkenntnisse über isolierte organisationale Variablen und ihre Determinanten zu gewinnen (Johnson/Duberley 2000, S. 8 f.). Die beschriebenen Kausalzusammenhänge sind zugleich Grundlage für eine planmäßige Umgestaltung der Organisation: Organisationale Beratung und Gestaltung wird als Formulierung und gezielter Transfer von Regeln und Verhaltensrichtlinien - etwa den Arbeitsablauf oder die Zentralisierung von Entscheidungsbefugnissen betreffend - verstanden. Der beratende Organisationsforscher nimmt nach diesem 
Modell mit den oberen Hierarchieebenen Kontakt auf, um sich der offiziellen Zielvorstellungen zu vergewissern, Fragen hinsichtlich des Forschungsdesigns zu klären und Bericht an die Auftraggeber zu erstatten. Unterstellt wird, dass die institutionelle Verwertung der Forschungsergebnisse die Planungssicherheit in der Organisation erhöht, Entscheidungsgrundlagen verbessert und eventuell Trendaussagen ermöglicht (Kubicek 1975, S. 15).

Diese Orientierung am naturwissenschaftlichen Ideal der exakten, empirischquantitativen Wissenschaft hat sicherlich zur Etablierung und Befestigung des Fachs beigetragen. Damals wie heute steht die zahlenmäßige Erfassung organisationaler Realität insbesondere für die Ziele der Generalisierung und der Objektivierung der gewonnen Erkenntnisse. Als geradezu prototypisch dafür lässt sich die von Frederick W. Taylor im frühen zwanzigsten Jahrhundert entwickelte Methode der wissenschaftlichen Betriebsführung (Scientific Management) nennen. Statt auf fehleranfällige „Spontaneinschätzungen“ und „Daumenregeln“ des Managements zu vertrauen, basierte das Scientific Management auf quantitativen Bestimmungen der Arbeitsproduktivität. Aus genau kontrollierten Messungen seien dann rationale Maßnahmen des Managements abzuleiten (vgl. Taylor 1967).

Diese frühen Ansätze der quantitativen Organisationsforschung beschränkten sich meistens auf die Untersuchung einer einzelnen Organisation. Dies mag damit zusammenhängen, dass die Entstehung der Organisationsforschung relativ stark als Praxisanwendung intendiert war. Der enge Bezug zur Betriebswirtschaftslehre, als positive Reflexionswissenschaft ihres Gegenstands, ist hier eindeutig. Aus dieser Perspektive sind vor allem einzelne Organisationen (Betriebe) von Interesse. Auch praxisbezogene Management- oder Betriebsvergleiche kommen im Grunde über diese Einzelperspektive noch kaum hinaus. Mit fortschreitender Theorieentwicklung löst sich die Organisationsforschung vom unmittelbaren Praxisbezug. Der Forschungszweck verlagert sich weg von Betriebszwecken hin zu Beiträgen der wissenschaftlichen Theoriebildung. Insbesondere in der Zeit nach dem Zweiten Weltkrieg bilden sich Ansätze aus, in denen mehrere Organisationen verglichen werden. Das Interesse verlagert sich von der Beforschung einzelner Organisationen hin zur vergleichenden Analyse mehrer Organisationen.

Die anwachsende Zahl an Organisationstheorien führte immer dringlicher die Frage vor Augen, was Organisationen eigentlich sind bzw. wie Organisationen angemessen zu beschreiben sind. Schon Max Weber (1976) hatte darauf mit der Beschreibung von Bürokratie als Herrschaftsapparat eine Antwort geliefert. Bis zur Nachkriegszeit waren aber, beispielsweise mit der verhaltenswissenschaftlichen Entscheidungstheorie nach Barnard (1938) und Simon (1947) oder mit herrschafts- und konflikttheoretischen Ansätzen (z. B. Selznick 1948), eine Reihe weiterer Forschungsrichtungen entstanden, die eine eigene Antwort versuchten. In Folge der theoriegeleiteten Frage nach dem Wesen oder auch Kernelementen von Organisation setzte sich die Forderung nach der vergleichenden Analyse von einer Vielzahl von Organisationen durch. Auch hier zeigte sich wiederum das Streben nach Generalisierung, die durch die Erhebung möglichst vieler Einzelfälle erreicht werden sollte. Wieder einmal waren quantifizierende Methoden gefragt. Insbesondere drei Forschungstraditionen motivierten das wachsende Interesse an quantifizierenden Vergleichen (vgl. Kubicek/Welter 1985, S. 3).

Ein erster Traditionsstrang fragte im Anschluss an die klassische Bürokratieanalyse von Weber, inwiefern der Idealtyp der bürokratischen Organisation auf weitere 
Organisationsarten, insbesondere Wirtschaftsbetriebe und Unternehmen, generalisiert werden könne. Im englischsprachigen Raum entwickelte sich nach der Übersetzung Max Webers „Wirtschaft und Gesellschaft“ ein breite Diskussion, ob die „rational-bürokratischen" Merkmale, wie Hierarchie, Regeln, geregelte Kompetenzen etc., beibehalten werden könnten (vgl. Weber 1968). Abweichungen von den Idealtypen waren empirisch evident. Allerdings war unklar, ob man die Idealtypen mit besseren Merkmalen ersetzen sollte oder ob man die Abweichungen von den Idealtypen als informelle Organisation zu beschreiben habe. Diese Diskussion generierte eine Reihe von Studien, die Webers Bürokratie-Typologien variierten.

Eine weitere Forschungstradition setzte mit der Entkolonialisierung nach dem Zweiten Weltkrieg ein. Es entwickelte sich ein wachsendes Interesse an empirischen Vergleichen zwischen Organisationen in traditionellen und nichttraditionellen Gesellschaften. Die Frage war unter anderem, ob Organisationsformen kulturspezifisch auftreten oder ob sich hier Universalien finden (Udy 1959, 1970).

Die dritte Forschungstradition entstand mit der englischsprachigen Managementlehre. Diese hatte - ganz in der Tradition von Taylor - lange Zeit versucht, universelle Organisationsprinzipien herauszuarbeiten. Solche Prinzipien sollten für jedes Unternehmen, jede Verwaltung, jedes Krankenhaus und jede Universität gleichermaßen Geltung besitzen. Es setzte sich jedoch die Erkenntnis durch, dass der „one best way“ für das Management von Organisationen nicht auszumachen ist. Die Entwicklung von Managementtheorien reagierte auf diese Einsicht unter anderem mit der Forderung, optimale Managementstile nach den spezifischen Situationen zu differenzieren, in denen sich die Organisationen jeweils befinden.

Diese Ausweitung der Organisationsforschung auf den analytischen Vergleich von mehreren Organisationen bedeutet eine Erweiterung der Forschungsperspektiven. Damit gelangte die Organisationsforschung in den Einzugsbereich weitere Wissenschaftsdisziplinen, etwa der Volkswirtschaftslehre und Politikwissenschaft, aber auch gesellschaftlicher, kultureller und psychologischer Perspektiven. Für die hier angebotene Übersicht ist festzuhalten, dass die quantitative Organisationsforschung beide Analyseschemata abdeckt: sowohl die Analyse einzelner Organisationen als auch die Untersuchung von Merkmalen einer ganzen Reihe von Organisationen.

Die Verwurzelung der Organisationsforschung in den quantitativen Methoden ist so stark, dass in manchen Disziplinen wie der Betriebswirtschaftslehre, der Organisationspsychologie oder der Arbeitswissenschaft die quantitativen Methoden eine dominierende Stellung, wenn nicht gar eine Monopolstellung einnehmen. In Anschluss an Frederick Taylor bildete sich eine eigene Schule, die unter dem Begriff „Work Study“ oder „Industrial Engineering“ Eingang in die Ingenieurswissenschaft, die Betriebswirtschaftslehre und die Arbeitswissenschaft fand. Die Arbeiten der Forschungsgruppe um Elton Mayo waren schließlich dafür verantwortlich, dass sich unter dem Begriff „Human Relations" eine eigene Forschungsrichtung ausbildete, die nach Wegen suchte mit einer stärkeren Fokussierung auf Gruppenprozesse sowohl die Arbeitszufriedenheit als auch die Produktivität zu erhöhen. Der Aufschwung der Organisationspsychologie und eines Teils der Arbeitswissenschaft ist durch die enorme Popularität dieses Ansatzes nach dem Zweiten Weltkrieg zu erklären. Die beibehaltene Orientierung der Human Relations Bewegung an zentralen quantifizierbaren Größen, wie der Produktivität, Zufriedenheitsscores etc., verdeutlicht dabei ihre starke Verbindung zu quantitativen Forschungsmethoden. 
Trotz der festen Verankerung in einer Reihe von organisationstheoretischen Ansätzen, hat die quantitative Methodik in der deutschsprachigen Organisationsforschung einen - im Vergleich zum englischsprachigen Sprachraum - eher geringen Stellenwert eingenommen. Dieser geringere Stellenwert lässt sich auch damit erklären, dass in den Großerhebungen, wie dem Sozio-oekonomischen Panel (SOEP), der allgemeinen Bevölkerungsumfrage in den Sozialwissenschaften (ALLBUS) und dem Wohlfahrtssurvey, kaum Daten erhoben werden, die etwas über die ,organisationsbezogene Strukturiertheit“ der Gesellschaft aussagen. Es liegen aus „erhebungs- und stichprobentechnischen Gründen“ lediglich partielle Informationen vor, beispielsweise über die Größe von Arbeitsorganisationen (Allmendinger/Hinz 2002, S. 23).

Ergebnis dieser mangelhaften Datenbestände ist, dass bei quantitativen Studien der Organisationsforschung häufig „selbstgenerierte Datenbestände“ benutzt werden. Eine Vielzahl dieser Erhebungen basiert auf Befragungen von Mitarbeitern mittels selbstgestalteter Fragebögen, die häufig nicht den wissenschaftlichen Minimalanforderungen genügen. Methodische Schwächen solcher Arbeiten wie geringe Stichprobenumfänge und Validitätsprobleme in der Datenerhebung sind evident.

Erst die immense Entwicklung des letzten Jahrzehnts im Bereich der preisgünstigen und zugleich leistungsfähigen Personalcomputer und entsprechender sozialwissenschaftlicher Software hat die Möglichkeiten quantitativer Forschung außerordentlich beflügelt. Eine Entwicklung, die durchaus vergleichbar zu dem Technologie-Push in den 60er Jahren verläuft (vgl. Kubicek/Welter 1985). Zunehmend anspruchsvollere Analysemethoden lassen sich jetzt auf breiterer Basis durchführen. Verbessert wurden damit aber nicht nur Analysemethoden. Im gleichen Maße profitieren davon Hypothesengenerierung und Theoriebildung, weil weitere empirische Zusammenhänge mit Hilfe der Computertechnologie analysiert, geprüft und überhaupt erst entdeckt werden können (vgl. insbesondere die Beiträge zur Inhalts-, zur Mehrebenen- und zur Netzwerkanalyse in diesem Band).

Ein weiterer Vorteil quantitativer Methoden, der ihre Bedeutung in der Organisationsforschung stärkt, ergibt sich aus ihrer Erwartungsindifferenz. Zwar fließen die subjektiven, möglichst theoretisch begründeten Erwartungen der Forscher durch Konzipierung und Operationalisierung im erheblichen Maße in den gewonnenen Datensatz ein. Liegen quantitative Daten aber erst einmal vor, sind sie relativ resistent gegen Erwartungszusammenhänge. Daraus resultieren bisweilen überraschende Ergebnisse in der Analyse oder in Replikationsstudien, die vorhandene Datensätze in weiteren Forschungskontexten nutzen. In diesem Sinne empfehlen sich quantifizierende Daten durchaus für Hypothesengenerierung und (Weiter-)Entwicklung von Theorien und öffnen so wissenschaftliche wie praxisorientierte Diskussionen über die Bedeutung und die Interpretation der gewonnenen Ergebnisse.

\section{Die Öffnung der Organisationsforschung für qualitative Methoden}

Die Verschiebung der Forschungsperspektive auf Organisationen als Sozialsysteme mit prinzipiell nicht-planbaren, dennoch aber spezifischen Interaktionen und zwischenmenschlichen Beziehungsformen hat die Bedeutung der quantitativen Methodik einschließlich ihres ingenieurwissenschaftlich geprägten Transfermodells relativiert. In bewusster Abgrenzung zum positivistischen Paradigma setzen Organisationswissenschaftler heute ver- 
mehrt auch qualitative Methoden ein, um das organisationale Geschehen aus der Sicht der handelnden Subjekte zu rekonstruieren, unerwartete Phänomene mit möglichst wenigen Vorentscheidungen hinsichtlich Design und Methode einzufangen und auf diese Weise menschliches Verhalten und Handeln einer prozessualen Sicht zugänglich zu machen. Ziel der qualitativen Forschung ist weniger der breit angelegte Vergleich organisationaler Wirkungsmechanismen als vielmehr das Eindringen in die Tiefe des Einzelfalls. Immer neue Details der jeweils untersuchten Einrichtung sollen den Blick für das „Unbekannte im scheinbar Bekannten“ freigeben, den Forscher mit „widerständigen“ und nicht-selektiven Daten konfrontieren und ihm so eine ganzheitliche, gegenstandsnahe Theoriebildung ermöglichen (Hopf 1993, S. 28; Silverman 1997).

Die zunehmende Verbreitung der qualitativen Methodik in der Organisationsforschung stützt sich auf zentrale Argumente der phänomenologischen Forschungstradition. Besondere Bedeutung messen qualitativ orientierte Organisationsforscher der frühen Erkenntnis der Klassiker bei, dass soziale Wirklichkeit nicht unabhängig von Zeit und Raum als objektive Wahrheit zu begreifen sei. Vielmehr wird sie als Ergebnis kollektiver und individueller Wahrnehmung und Interpretation betrachtet und dementsprechend prozessual, d. h. in Form von Kommunikations- oder Handlungssequenzen im alltäglichen Kontext untersucht (Glaser/Strauss 1993, S. 92 f.). Aufgabe des Empirikers ist daher nicht die Isolierung einzelner Kausalitäten, sondern die Rekonstruktion subjektiv gemeinten Sinns und das „Verstehen“ komplexer Zusammenhänge (vgl. Schütz 1971). Auch gilt die subjektive Wahrnehmung des Forschers nicht als Störquelle, sondern als selbstverständlicher Bestandteil des Forschungsprozesses. Der Wissenschaftler ist aus phänomenologischer Sicht selbst in die Deutungs- und Interaktionsprozesse der Organisation eingebunden. Da seine Forschungsergebnisse beständig neue Wirklichkeitskonstruktionen und Bedeutungszusammenhänge kreieren, nimmt er unweigerlich an der Konstituierung seines Forschungsgegenstandes teil (Flick/Kardorff/Steinke 2000, S. 23). Forschung und Beratung fallen schließlich dort in eins, wo Konzept- und Methodenentwicklung im dialogischen Vorgehen den Bedürfnissen der betroffenen Mitarbeiter angepasst werden und verschiedene Rückkopplungsschleifen im Forschungsprozess (z. B. in Form von Gruppendiskussionen) einen gemeinsamen Lernprozess von Forschern und Organisationsmitgliedern ermöglichen (Guba/Lincoln 1989, S. 42).

Wo liegen die Gemeinsamkeiten der verschiedenen qualitativen Methoden der Organisationsforschung? Als prägendes Merkmal ist zunächst das Prinzip der Offenheit hervorzuheben, das sich in sämtlichen Phasen des Forschungsprozesses bemerkbar macht. Um eine Nähe zum Gegenstand herzustellen, setzen qualitativ orientierte Organisationsforscher in der Phase der Erhebung unstandardisierte oder wenig standardisierte Instrumente ein (siehe die Beiträge zum Experteninterview, zur teilnehmenden Beobachtung, aber auch zur visualisierten Diskussionsführung in diesem Band). Ausgewertet werden die Ergebnisse „verdichtend“ oder „typisierend“ beispielsweise im Rahmen einer Fallstudie oder eines Erfahrungsberichts; verallgemeinernde Vergleiche finden sich, wenn überhaupt, erst in den Abschlussphasen der Untersuchung (Hopf 1993, S. 14; Kitay/Callus 1998, S. 101 ff.). Als zweites Kennzeichen sei auf die Gegenstandsangemessenheit der Verfahren hingewiesen. Entscheidend für deren Auswahl und Bewertung ist nicht die statistisch zu ermittelnde „Messgenauigkeit“, sondern der untersuchte Gegenstand, seine Eigenheiten, sein alltäglicher Kontext sowie die besondere Fragestellung der Studie. Eine Weiterentwicklung des vorhandenen Methodeninstrumentariums geht daher häufig auf 
inhaltliche Überlegungen zurück (Flick/Kardorff/Steinke 2000, S. 22 f.; klassisch Strauss 1991). Schließlich geben auch die Gütekriterien der qualitativen Forschung Aufschluss über die Eigentümlichkeit ihrer Instrumente und Verfahren. Aufgrund ihres gegenstandsund kontextabhängigen Charakters entzieht sich die qualitative Forschung einer Beurteilung nach den klassischen Kriterien der quantitativ-hypothesentestenden Wissenschaft. An die Stelle der exakten Überprüfung von Validität, Repräsentativität und Reliabilität tritt ein bewusst flexibel gehaltenes System von Kriterien, das der geringen Formalisierbarkeit und Standardisierbarkeit der Forschungsaktivitäten und -instrumente Rechnung trägt. $\mathrm{Zu}$ den am häufigsten genannten Kriterien gehört hier die „Nachvollziehbarkeit“, die über ausführliche Dokumentation, interkollegiale Kontrollen und kodifizierte Vorgehensweisen verbessert werden soll (Lincoln/Guba 1985, S. 292; Steinke 2000, S. 323 f.).

Eine gewisse Vorrangstellung unter den vielfältigen Verfahren, die mittlerweile im Bereich der Organisationsanalyse Verwendung gefunden haben, kommt dem qualitativen Interview zu. Ihren ersten Durchbruch hatte die offene Befragung bereits im Rahmen der berühmten Hawthorne-Studien, in deren Verlauf die Wissenschaftler auf unstandardisierte mündliche Interviews umschwenkten, um die Relevanz und Authentizität ihrer Ergebnisse zu erhöhen (Roethlisberger/Dickson 1939; vgl. Rosenstiel 2000b, S. 232). Heute wird insbesondere das leitfadengestützte Experteninterview eingesetzt, um das „Fach-, Dienst- und Geheimwissen“ der Professionellen gezielt für explorative wie auch für hypothesenprüfende Forschungsfragen nutzen zu können (Pfaff/Bentz 1998, S. 315; siehe den Beitrag zum Experteninterview in diesem Band). In Forschungsprojekten mit Interventionsabsichten hat außerdem das Gruppeninterview bzw. die Gruppendiskussion einen festen Platz. Die freie oder themenzentrierte Reflexion unter ausgewählten Organisationsmitgliedern hat sich vor allem in den Phasen der Maßnahmenplanung und -evaluation als hilfreich erwiesen (Rosenstiel 2000a; Guba/Lincoln 1989; siehe den Beitrag zur Gruppendiskussion in diesem Band). Schließlich bedient sich die empirische Organisationsforschung überdurchschnittlich häufig der Dokumenten- und Aktenanalyse, z. B. wenn sie sich in der Phase der Organisationsdiagnose mittels interner Schriftstücke (Aktennotizen, Verträge, Tätigkeitsbeschreibungen usw.) den Strukturen und Prozessen einer Einrichtung zu nähern versucht oder im Rahmen einer Evaluation deren Wirksamkeit bewertet (Phillips/Palfrey/Thomas 1994, S. 46 f.).

Methodische Innovationen erwarten Organisationswissenschaftler derzeit unter anderem von der breiteren Anwendung bislang „untergenutzter“ unstandardisierter Verfahren. Die inhaltliche Schwerpunktverlagerung der empirischen Forschung auf soziale Phänomene im Alltagsgeschehen der Organisation, auf die Interaktionen, Praktiken und Diskurse der Organisationsmitglieder, erzeugt einen Bedarf an „naturalistischen“ und „kontextnahen“ Daten, wie sie mit Hilfe offener und unstandardisierter Instrumente wie z. B. des narrativen Interviews oder der teilnehmenden Beobachtung gewonnen werden können. Die vergleichsweise aufwendigen Datenerhebungs- und Auswertungsphasen wiegen diese Verfahren durch ihre Nähe zum konkreten Handlungsgeschehen und durch ihren Zugang zum impliziten Wissenspotenzial sowie zu den Interpretationen und Bewertungen der Interaktionsteilnehmer bei weitem auf (Brewerton/Millward 2001, S. $11 \mathrm{f}$; Becker/Geer 1993, S. 140). So gilt als besonderer Vorzug der teilnehmenden Beobachtung die fast perfekte Auflösung der Barrieren zwischen Forscher und Beobachtungsfeld: Im unmittelbaren Kontakt mit den untersuchten Personen ist der Wissenschaftler in der Lage, neben den hypothetisch vorausgesetzten, auch vollkommen unerwartete Einsichten in das 
natürliche und situationsspezifische Verhalten der Teilnehmer zu sammeln (Friedman/McDaniel 1998; siehe den Beitrag über teilnehmende Beobachtung in diesem Band). In ähnlicher Weise enthüllt das biographisch-narrative Interview, das sich zugunsten des Erzählprinzips vom strikten Frage-Antwort-Schema gelöst hat, vergleichsweise effektiver als standardisierte Interviewformen die im Einzelfall hochkomplexen Prozesse der Bedeutungs- und Sinnherstellung, der Produktion von Selbst- und Weltbildern in der Organisation (Miller/Glassner 1997; siehe den Beitrag über narrative Interviews in diesem Band). Neben diesen offenen Formen der Beobachtung und Befragung ist auch die weit verbreitete Dokumentenanalyse mit Blick auf „sozial organisierte Praktiken der Produktion und Rezeption" rund um den Prozess der internen Verschriftlichung in ihren Potenzialen noch lange nicht ausgeschöpft. Die interpretative Auswertung „latenter“ Kommunikationsinhalte, beispielsweise durch eine qualitative Inhaltsanalyse, ist bislang zugunsten einer faktenorientierten Vorgehensweise weitgehend vernachlässigt worden (Wolff 2000, S. 505; Watson 1997; siehe den Beitrag über Artefaktanalyse in diesem Band).

Innovationen werden jedoch nicht nur von einem ,mutigeren“ Umgang mit weitgehend unstandardisierten Verfahren, sondern auch von einer allgemeinen Flexibilisierung im Zuschnitt der Forschungsstrategien erwartet. Als Ausdruck einer zunehmend experimentellen und unorthodoxen Haltung in Fragen des Forschungsdesigns wie auch der Rolle des Forschers im Untersuchungsfeld kann hier etwa der Zuwachs an ethnographischen Studien in der Organisationsforschung gewertet werden (vgl. z. B. Helmers 1993; Neuberger/Kompa 1987). Als deren wesentliches Kennzeichen gilt im Allgemeinen der Fokus auf das soziale Geschehen, die kommunikative und interaktive Ordnung in abgrenzbaren Gruppen. Die ethnographische Forschung konzentriert sich auf die in der Organisation vorherrschenden Formen der Sinngebung und Verständigung und steht damit stets auch in enger Verbindung zur phänomenologischen Lebensweltanalyse (Flick 2001, S. 57 f.; Hirschauer/Amann 1997). Unabdingbar für die Untersuchung alltäglich angewendeter und modifizierter Interaktions- und Kommunikationsregeln (so genannter Ethnomethoden nach Garfinkel 1967) ist dem Ethnographen dabei die „Kopräsenz von Beobachter und Geschehen“. Der über einen längeren Zeitraum andauernde Feldaufenthalt schließt den Mitvollzug sozialer Ereignisse, die Übernahme einer allseits akzeptierten Rolle und den Aufbau von Vertrauensbeziehungen zu Untersuchungspersonen ein. Der Forscher entscheidet situations- und fallangemessen über den Einsatz der geeigneten Methodik und berichtet nachträglich in Feldprotokollen über seine Erfahrungen. Dabei haben gerade die nicht-planbaren, zufälligen und individuellen Momente des Forschungsprozesses der Ethnographie das Kompliment der ,art of fieldwork“ eingebracht, aber auch den kritischen Vergleich mit journalistischen Techniken provoziert (Lüders 2000, S. 391 ff.; Wolcott 1995). Im Bereich der Organisationswissenschaften wurde die ethnographische Vorgehensweise zuerst in der Arbeits- und Berufsforschung angewendet. So untersuchten bereits die klassischen Studien der 1950er Jahre Veränderungen im Zuschnitt der Arbeitstätigkeiten, im Ausmaß der professionellen Autonomie oder der Organisierbarkeit von Klasseninteressen mittels teilnehmender Beobachtungen und offener Befragungen (vgl. Wilensky 1956; Sayles/Strauss 1953; siehe als Überblick z. B. Friedman/McDaniel 1998). Heute greifen vor allem Forschungen zur Organisationskultur auf die ethnographische Vorgehensweise zurück, wenn sie sich beispielsweise den Zeremonien und Ritualen, den Mythen oder Tabus einer Organisation als deren Symptom bzw. Objektivierung nähern (Kieser 1988; Neuberger 1995). 


\section{Zeitliche, sachliche und soziale Arbeitsteilung und weitergehende Kombinationsmöglichkeiten von quantitativen und qualitativen Methoden}

Die Forderung, quantitative und qualitative Methoden zu kombinieren, wird in der Organisationsforschung seit längerem laut. Die strikte Trennung zwischen den wissenschaftlichen Paradigmen quantitativer und qualitativer Methodik wird als unfruchtbar und beengend beklagt. Tatsächlich bestehen aber Formen der zeitlichen, sachlichen oder sozialen Arbeitsteilung, die die Methodenkombination problematisch werden lassen.

Bei einer zeitlichen Arbeitsteilung wird der Forschungsprozess in verschiedene Phasen unterteilt, die dann mit unterschiedlichen qualitativen und quantitativen Methoden bearbeitet werden. In einer Variante werden zu Beginn der Untersuchung statistisch auswertbares Material erhoben, zur Unterstützung der Interpretation dann aber punktuell qualitative Interviews geführt (vgl. die Hawthorne-Experimente, Mayo 1933). In einer anderen Variante wird zur Vorbereitung einer qualitativen Untersuchung erst eine repräsentative Stichprobe gezogen. Dadurch sollen Aussagen darüber getroffen werden, inwieweit die Stichprobe der qualitativen Untersuchung bezüglich bestimmter Merkmale typisch ist (vgl. Freter/Hollstein/Werle 1992, S. 98; Merkens 1997, S. 97 ff.). Bei einer besonders von quantitativen Organisationsforschern bevorzugte Variante werden qualitative Interviews geführt, um ein Gespür für das Feld zu bekommen und die Hypothesen zu entwickeln. In der „eigentlichen“ Untersuchung werden dann quantitative Methoden herangezogen, um die gewonnenen Hypothesen zu testen und die Erkenntnisse zu sichern (Barton/Lazarfeld 1955, S. 321 ff.). Gerade die letzte Form der Arbeitsteilung wird jedoch immer häufiger umgangen. Nicht nur erheben qualitative Methoden zunehmend den Anspruch zur Überprüfung von Hypothesen geeignet zu sein, sondern es werden immer mehr quantitative Methoden für die Generierung von Hypothesen entwickelt. Gerade die Faktorenanalyse und die Clusteranalyse eigenen sich hervorragend dazu, Zusammenhänge zu erschließen und Hypothesen zu generieren (vgl. Hollstein/Ullrich 2003, S. 33).

Bei der sachlichen Arbeitsteilung werden thematische Forschungsfelder so definiert und damit getrennt, dass die einen mit quantitativen Methoden und die anderen mit qualitativen Methoden bearbeitet werden. Diese sachliche Trennung resultiert häufig in einer Trennung sogenannter mikro- und makrosozialer Phänomene. Markoanalysen, wie beispielsweise der Strukturenvergleich mehrerer Organisationen, tendieren auf Grund der möglichst hohen Fallzahlen zur Anwendung quantitativer Forschungsdesigns. Aus der Perspektive einer quantitativ orientierten Sozialforschung können bei den Vergleichen zwischen Organisationen individuelle Interpretationen vernachlässigt werden. Bei der Messung der Formalstruktur von Organisationen handelte es sich um weitgehend verobjektivierte Sachverhalte. Wahrnehmungsunterschiede im Detail scheinen dafür nicht von großer Bedeutung. Dagegen eigenen sich für Mikroanalysen, beispielsweise bei der Wiedergabe mikropolitischer Prozesse in einer teilautonomen Arbeitsgruppe, eher qualitative Methoden.

Als dritte Form ist die soziale Arbeitsteilung anzuführen. Bei dieser weitverbreiteten Arbeitsteilung werden qualitative und quantitative Untersuchungen von verschiedenen Forschern wahrgenommen. Zumeist spezialisieren sich Forscher auf bestimmte Methoden und Forschungstraditionen, die geradezu identitätsstiftend wirken. Die Arbeitsteilung stabilisiert sich dann an den wissenschaftlichen Identitäten der im Forschungsprozess beteiligten Personen. 
In der empirischen Organisationsforschung wie auch allgemein in der empirischen Sozialforschung macht sich eine zunehmende Unzufriedenheit mit diesen Formen der zeitlichen, sachlichen und sozialen Arbeitsteilung breit (vgl. Engler 1997, S. 123 f.). Insbesondere die Forderung, die strikte Differenzierung zwischen quantitativen und qualitativen Forschungsansätzen zu überwinden, scheint inzwischen schon zum Standardrepertoire methodischer Abhandlungen zu gehören. Unter Begriffen wie Methoden-Mix, multimethodischem Vorgehen (vgl. Campbell/Fiske 1959) oder Triangulation (Kelle/ Erzberger 2000) setzen sich zunehmend Verfahren durch, die bei der Untersuchung auf mehrere Methoden zurückgreifen. Der Begriff der Triangulation stammt ursprünglich aus der Navigations- und Militärlehre. Dort wird der Einsatz von multiplen Bezugspunkten dafür genutzt, die genaue Position eines Objektes zu bestimmen (vgl. Jick 1979, S. 24). Analog dazu erhofft man sich durch die Anwendung mehrerer methodischer Bezugspunkte Erklärungskraft und Realitätsbezug wissenschaftlicher Forschungen zu erhöhen (vgl. Kelle/ Erzberger 2000).

\section{Der Verwendungsbezug der Organisationsforschung außerhalb der Wissenschaft}

Die Organisationsforschung hat, wie bereits angedeutet, Phasen eines sozialtechnologisch geprägten Verständnisses von Beratung und Gestaltung hinter sich, in denen vorzugsweise betriebswirtschaftliches oder sozialpsychologisches Expertenwissen „rezeptartig“ aufbereitet und in Praxiszusammenhänge transferiert wurde (vgl. den Überblick bei Kieser 1995, S. 28 ff.; 86 ff.; 107 ff.). Das Scheitern vieler technokratischer Interventionsprojekte, in Deutschland beispielsweise im Gefolge des staatlich geförderten Programms zur „Humanisierung der Arbeit“, war der Grund dafür, dass sich bedeutende Stränge der Organisationsforschung (Ausnahmen sind die Betriebswirtschaftslehre sowie Teile der Arbeitswissenschaft und der Organisationspsychologie) einer zu eng geführten Verwendung ihres Wissens entzogen (vgl. z. B. Braczyk 1992). Unkontrollierbare Interessenkonstellationen im Interventionsprozess, unbeeinflussbare Randbedingungen oder auch der nur strategische und instrumentelle Umgang mit dem Expertenwissen in der Organisation hatten eine Krise des einfachen, ingenieurwissenschaftlich geprägten Transfermodells ausgelöst und spätestens ab Mitte der 1980er Jahre einen Rückzug auf Fragen des wissenschaftlichen Ertrags und Erkenntnisgewinns empirischer Forschungsprojekte eingeleitet. „Gestaltung durch Aufklärung“ hieß das defensive Programm deutscher Organisationswissenschaftler und Industriesoziologen, mit dem außerwissenschaftliche, praktische Beratungsaufgaben zunächst hinter die analytisch-diagnostischen Fähigkeiten der Disziplin zurückgestellt wurden.

In den 1990er Jahren öffnete sich die Organisationsforschung wieder in einem breiteren Ausmaß für den Transfer ihrer Ergebnisse in die Organisationspraxis. Gegründet war diese erneute Öffnung auf die Überzeugung, dass nicht von einer simplen Übertragung „richtiger“ wissenschaftlicher Erkenntnisse in eine „unterbelichtete“ Praxis auszugehen sei, sondern dass die Handlungsweisen und Formen der Wissensgenerierung in Unternehmen, Verbänden, Verwaltungen und Krankenhäusern vielmehr in ihrer Verschiedenheit anzuerkennen seien. Wissenschaftliches Expertenwissen wird den Problemlösungskompetenzen der Praktiker nicht länger hierarchisch übergeordnet, es wird ihnen als Fremdwahrnehmung und alternative Deutungsweise gleichrangig beigestellt. 
Dabei haben die Betonung der Problemlösungskompetenzen in der Organisation und die vorläufige Herabsetzung des ,zweiten Blicks“ des Organisationsforschers und/oder des Organisationsberaters auf das Niveau eines verzichtbaren Luxus ihren guten Grund. Eine Organisation kann ohne die Handlungen der Organisationsmitglieder, d. h. ohne den „ersten Blick“ der Organisationspraktiker, nicht auskommen - die Manager würden nicht entscheiden, die Mitarbeiter nicht produzieren und der Außendienst nicht verkaufen. Ob und inwieweit der „Zweite Blick“ für die Organisation sinnvoll ist, hängt von der Situation ab. Ein Zuviel an Reflexion kann die Organisation verwirren und ihre Handlungen blockieren (siehe Brunsson 1985).

Auch andere Wissenschaftszweige und Beratergruppen haben mittlerweile die Rückspiegelung von Forschungsergebnissen als einen Prozess mit eigenen Gesetzmäßigkeiten, Funktionsweisen und Tücken in den Mittelpunkt gerückt. Der amerikanischen Evaluationsund Verwendungsforschung beispielsweise entstammt die Erkenntnis, dass Forschungsergebnisse in zeitlich und räumlich versetzten Interpretationsprozessen klein gearbeitet und in Abhängigkeit von kommunikativen Routinen und beruflichen Traditionen reformuliert werden, bis sie schließlich die Form von Planungs- und Steuerungswissen in der Organisation annehmen (Weiß 1987; siehe auch Beck/Bonß 1989, S. 22 f.). Die vorwiegend im deutschsprachigen Raum etablierte systemische Beratung hat herausgestellt, dass das Verhältnis zwischen Forschern bzw. Beratern und Organisation als Kontakt zweier verschiedener Systeme begriffen werden muss. Im Verlauf des Kontakts bilden sich „Beratungssysteme“ und „Wissenschafts-Praxis-Kontaktfelder“" heraus, mit deren Hilfe die Kommunikation zwischen den beiden selbstreferenziellen Systemen mühevoll aufrechterhalten wird (vgl. z. B. Wimmer 1993; Mingers 1996). Ansätze der organisationalen Beratung, die an die theoretischen Überlegungen zu Grenzen der Zweckrationalität anknüpfen, betonen, dass die in jeder Organisation existierenden blinden Flecken unmöglich im Sinne einer „Aufklärung der Organisation über sich selbst“" aufgedeckt werden können. Forscher und Berater müssen vielmehr eigene Interventionspraktiken entwickeln, um das Wahrnehmungsspektrum der Organisation zu erweitern.

Ob diese (erneute) Öffnung der Organisationsforschung in Richtung Organisationspraxis zu einer sichtbaren „Verbesserung“ von Management und Beratung geführt hat bzw. künftig führen wird, lässt sich noch nicht abschätzen. Deutlich ist jedoch, dass umgekehrt eine auffällige Bereicherung des Methodenspektrums in der empirischen Organisationsforschung stattgefunden hat. So wurden die in den letzten Jahren zunehmend etablierten gruppenorientierten Erhebungsmethoden (Organisationskarten, visualisierte Diskussionsführung, Open Space, Rollenspiel u. a.; siehe die entsprechenden Beiträge in diesem Band) aus Organisationsberatung, Personalentwicklung oder Aus- und Weiterbildung übernommen und als Methode der empirischen Sozialforschung weiterentwickelt. Es ist interessant, dass ihre Wurzeln häufig in der frühen Organisationsforschung liegen (z. B. der Human-Relations-Bewegung in der Tradition Kurt Lewins), dass sie aber im wissenschaftlichen Kontext lange Zeit kaum beachtet wurden. Ihren verstärkten Einsatz in der neueren Organisationsforschung kann man daher auch als Wiederentdeckung früherer Forschungstraditionen verstehen. Eine neue Reflexivität im Zuschnitt der Forschungsstrategien ergänzt diese Methodenvielfalt: Im Mittelpunkt auch wissenschaftlicher Forschungsprojekte stehen immer häufiger die von Organisationsmitgliedern wahrgenommenen praktischen Probleme und Handlungszwänge, der Dialog zwischen Forschern und beforschten Einrichtungen sowie die Entwicklung eines organisations- und problemspezifischen Methodenmixes. 
Forschung wird als sozialer Lernprozess begriffen, in dessen Verlauf nicht nur die Verwissenschaftlichung des Praxisfeldes intendiert ist, sondern auch Rückwirkungen auf Fragestellungen, Methoden und Standards der Forschung zugelassen sind (Beck/Bonß 1989, S. 33 f.).

Zusammenfassend lässt sich feststellen, dass drei Entwicklungen die Methodendiskussion der Organisationsforschung maßgeblich beeinflusst haben: der Abschied von zweckrationalen Vereinfachungen im Organisationsverständnis, der überhaupt erst Raum für eine stärkere Betonung qualitativer Methoden geschaffen hat; die Ausdifferenzierung und vielfältige Entwicklung der empirischen Sozialforschung als Fundament der organisationswissenschaftlichen Methodendebatte; schließlich die zunehmende Problematisierung des Transfermodells, die zu einer Öffnung der wissenschaftlichen Forschung für Methoden aus den Bereichen Beratung, Personalentwicklung und Weiterbildung geführt hat. Die weitere Methodendiskussion in der Organisationsforschung wird unter anderem davon abhängen, inwieweit es gelingt, diese drei Stränge zusammenzuführen.

\section{Literatur}

Allmendinger, Jutta/Hinz, Thomas (2002): Perspektiven der Organisationssoziologie, in: dies. (Hrsg.), Organisationssoziologie. Sonderheft 42 der Kölner Zeitschrift für Soziologie und Sozialpsychologie, Wiesbaden, S. 9-28

Barnard, Chester I. (1938): The Functions of the Executive, Cambridge

Barton, Allen H./Lazarsfeld, Paul F. (1955): Some Functions of Qualitative Analysis in Social Research, in: Frankfurter Beiträge zur Soziologie, 1, S. 321-361

Barton, Allen H./Lazarsfeld, Paul F. (1993): Das Verhältnis von theoretischer und empirischer Analyse im Rahmen qualitativer Sozialforschung, in: Christel Hopf/Elmar Weingarten (Hrsg.), Qualitative Sozialforschung, Stuttgart, S. 41-89

Beck, Ulrich/Bonß, Wolfgang (1989): Verwissenschaftlichung ohne Aufklärung? Zum Strukturwandel von Wissenschaft und Praxis, in: dies. (Hrsg.), Weder Sozialtechnologie noch Aufklärung? Analysen zur Verwendung sozialwissenschaftlichen Wissens, Frankfurt a. M., S. 7-45

Becker, Howard S./Geer, Blanche (1993): Teilnehmende Beobachtung: Die Analyse qualitativer Forschungsergebnisse, in: Christel Hopf/Elmar Weingarten (Hrsg.), Qualitative Sozialforschung, Stuttgart, S. 139-166

Blau, Peter M. (1955): The Dynamics of Bureaucracy, Chicago

Braczyk, Hans-Joachim (1992): Die Qual der Wahl. Optionen der Gestaltung von Arbeit und Technik als Organisationsproblem, Berlin

Brewerton, Paul M./Millward, Lynne J. (2001): Organizational Research Methods, London

Brunsson, Nils (1985): The Irrational Organization. Irrationality as a Basis for Organizational Action and Change, Chichester u. a.

Campbell, Donald T./Fiske, Donald W. (1959): Convergent and Discriminant Validation by the Multitrait-multimethod Matrix, in: Psychological Bulletin, 56, S. 81-105

Crozier, Michel/Friedberg, Erhard (1979): Macht und Organisation. Die Zwänge kollektiven Handelns, Königstein/Ts.

Dierkes, Meinolf/Rosenstiel, Lutz v./Steger, Ulrich (Hrsg.) (1993): Unternehmenskultur in Theorie und Praxis, Frankfurt a. M.

Engler, Steffani (1997): Zur Kombination von qualitativen und quantitativen Methoden, in: Barbara Friebertshäuser/Annedore Prengel (Hrsg.), Handbuch Qualitative Forschungsmethoden in der Erziehungswissenschaft, München, S. 118-130 
Flick, Uwe (2001): Qualitative Sozialforschung - Stand der Dinge, in: Soziologie, 2, S. 53-66

Flick, Uwe/Kardorff, Ernst v./Steinke, Ines (2000): Was ist qualitative Forschung? Einleitung und Überblick, in: dies. (Hrsg.), Qualitative Forschung. Ein Handbuch, Reinbek bei Hamburg, S. $13-29$

Freter, Hans-Jürgen/Hollstein, Betina/Werle, Markus (1992): Integration qualitativer und quantitativer Verfahrensweisen - Methodologie und Forschungspraxis, in: ZUMA-Nachrichten, 29/1992, S. 98-114

Friedberg, Erhard (1993): Le pouvoir et la règle. Dynamiques de 1'action organisée, Paris

Friedman, Raymond A./McDaniel, Darren C. (1998): In the Eye of the Beholder: Ethnography in the Study of Work, in: Keith Whitfield/George Strauss (Hrsg.), Researching the World of Work, New York, S. 113-126

Garfinkel, Harold (1967): Studies in Ethnomethodology, Englewood Cliffs NJ

Glaser, Barney G./Strauss, Anselm L. (1967): The Discovery of Grounded Theory, Chicago

Glaser, Barney G./Strauss, Anselm L. (1993): Die Entdeckung gegenstandsbezogener Theorie: Eine Grundstrategie qualitativer Sozialforschung, in: Christel Hopf/Elmar Weingarten (Hrsg.), Qualitative Sozialforschung, Stuttgart, S. 91-111

Guba, Egon G./Lincoln, Yvonna S. (1989): Fourth Generation Evaluation, Newbury Park et al.

Hall, Richard H. (1963): The Concept of Bureaucracy, in: American Journal of Sociology, 69, S. $32-40$

Helmers, Sabine (1993): Ethnologie der Arbeitswelt. Beispiele aus europäischen und außereuropäischen Feldern, Bonn

Hirschauer, Stefan/Amann, Klaus (Hrsg.) (1997): Die Befremdung der eigenen Kultur. Zur ethnographischen Herausforderung soziologischer Empirie, Frankfurt a. M.

Hollstein, Betina/Ulrich, Carsten G. (2003): Einheit trotz Vielfalt? Zum konstitutiven Kern qualitativer Forschung, in: Soziologie, 32, S. 29-43

Hopf, Christel (1993): Soziologie und qualitative Sozialforschung, in: Christel Hopf/Elmar Weingarten (Hrsg.), Qualitative Sozialforschung, Stuttgart, S. 11-37

Jick, Todd D. (1979): Mixing Qualitative and Quantitative Methods: Triangulation in Action, in: Administrative Science Quarterly, 24, S. 602-611

Johnson, Phil/Duberley, Joanne (2000): Understanding Management Research, London

Kelle, Udo/Erzberger, Christian (2000): Qualitative und quantitative Methoden: kein Gegensatz, in: Uwe Flick/Ernst von Kardorff/Ines Steinke (Hrsg.), Qualitative Forschung. Ein Handbuch, Reinbek, S. 299-308

Kieser, Alfred (1988): Von der Morgenansprache zum „Gemeinsamen HP-Frühstück“. Zur Funktion von Werten, Mythen, Ritualen und Symbolen, in: Eberhard Dülfer (Hrsg.), Organisationskultur, Stuttgart, S. 207-225

Kieser, Alfred (1995): Organisationstheorien, 2. Auflage, Stuttgart et al.

Kieserling, André (1993): Konturen einer soziologischen Unternehmensberatung, unveröff. Manuskript, Bielefeld

Kieserling, André (1994): Organisationssoziologie und Unternehmensberatung. 6 Lehrvorträge, unveröff. Manuskript, Bielefeld

Kitay, Jim/Callus, Ron (1998): The Role and Challenge of Case Study Design in Industrial Relations Research, in: Keith Whitfield/George Strauss (Hrsg.), Researching the World of Work, New York, S. 101-112

Kubicek, Herbert (1975): Empirische Organisationsforschung, Stuttgart

Kubicek, Herbert/Welter, Günter (1985): Messung der Organisationsstruktur, Stuttgart

Kühl, Stefan (2000a): Grenzen der Vermarktlichung. Die Mythen um unternehmerisch handelnde Mitarbeiter, in: WSI-Mitteilungen, 53, S. 818-828

Kühl, Stefan (2000b): Das Regenmacher-Phänomen. Widersprüche und Aberglaube im Konzept der lernenden Organisation, Frankfurt a. M./New York

Lincoln, Yvonna S./Guba, Egon G. (1985): Naturalistic Inquiry, Beverly Hills 
Lüders, Christian (2000): Beobachten im Feld und Ethnographie, in: Uwe Flick/Ernst von Kardorff/Ines Steinke (Hrsg.), Qualitative Forschung. Ein Handbuch, Reinbek bei Hamburg, S. $384-401$

Luhmann, Niklas (1973): Zweckbegriff und Systemrationalität. Über die Funktion von Zwecken in sozialen Systemen, Frankfurt a. M.

Luhmann, Niklas (1995): Funktionen und Folgen formaler Organisation, 4. Auflage, Berlin

Luhmann, Niklas (1997): Die Gesellschaft der Gesellschaft, Frankfurt a. M.

Luhmann, Niklas (2000): Organisation und Entscheidung, Opladen

March, James G. (1990): Entscheidung und Organisation: Kritische und konstruktive Beiträge, Entwicklungen und Perspektiven, Wiesbaden

Mayo, Elton (1933): The Human Problems of an Industrial Civilization, Boston

Merkens, Hans (1997): Stichproben bei qualitativen Studien, in: Barbara Friebertshäuser/Annedore Prengel (Hrsg.), Handbuch Qualitative Forschungsmethoden in der Erziehungswissenschaft, München, S. 97-106

Merton, Robert K. (1957): Social Theory and Social Structure, 2. Auflage, Glencoe

Miller, Jody/Glassner, Barry (1997): The „Inside“ and the „Outside“: Finding Realities in Interviews, in: David Silverman (Hrsg.), Qualitative Research. Theory, Method and Practice, London et al., S. $99-112$

Mingers, Susanne (1996): Systemische Organisationsberatung. Eine Konfrontation von Theorie und Praxis, Frankfurt a. M./New York

Neuberger, Oswald (1995): Mikropolitik. Der alltägliche Aufbau und Einsatz von Macht in Organisationen, Stuttgart

Neuberger, Oswald/Kompa, Ain (1987): Wir, die Firma, Weinheim

Pfaff, Holger/Bentz, Joachim (1998): Subjektive Daten - objektive Analyse, in: Friedrich W. Schwartz et al. (Hrsg.), Das Public Health Buch. Gesundheit und Gesundheitswesen, München, S. $310-328$

Phillips, Ceri/Palfrey, Colin/Thomas, Paul (1994): Evaluating Health and Social Care, London

Pugh, Derek S./Hickson, David J. (1976): Organizational Structure in its Context. The Aston Programme I, Westmead

Roethlisberger, Fritz Jules/Dickson, William J. (1939): Management and the Worker. An Account of a Research Program Conducted by the Western Electric Company, Hawthorne Works, Chicago, Cambridge Mass.

Rosenstiel, Lutz v. (2000a): Grundlagen der Organisationspsychologie, Stuttgart

Rosenstiel, Lutz v. (2000b): Organisationsanalyse, in: Uwe Flick/Ernst von Kardorff/Ines Steinke (Hrsg.), Qualitative Forschung. Ein Handbuch, Reinbek bei Hamburg, S. 224-238

Sayles, Leonard R./Strauss, George (1953): The Local Union, New York

Schnelle, Wolfgang (2001): Moderieren von Verständigungsprozessen, Quickborn

Schreyögg, Astrid (1992): Supervision. Ein integratives Modell, Paderborn

Schütz, Alfred (1971): Gesammelte Aufsätze. Bd. 1: Das Problem der sozialen Wirklichkeit, Den Haag

Selznick, Philip (1948): Foundations of the Theory of Organisations, in: American Sociological Review, 13, 1, S. 25-35

Silverman, David (1997): Introducing Qualitative Research, in: ders. (Hrsg.), Qualitative Research. Theory, Method and Practice, London u. a., S. 1-7

Simon, Herbert A. (1947): Administrative Behavior, New York

Simon, Herbert A. (1976): Administrative Behavior. A Study of Decision-Making Processes in Administrative Organizations, 3. Auflage, New York

Steinke, Ines (2000): Gütekriterien qualitativer Forschung, in: Uwe Flick/Ernst von Kardorff/Ines Steinke (Hrsg.), Qualitative Forschung. Ein Handbuch, Reinbek bei Hamburg, S. 319-331

Strauss, Anselm L. (1991): Grundlagen qualitativer Sozialforschung - Datenanalyse und Theoriebildung in der empirischen soziologischen Forschung, München

Tacke, Veronika (2001) (Hrsg.), Organisation und gesellschaftliche Differenzierung, Wiesbaden 
Taylor, Frederick W. (1967): The Principles of Scientific Management, New York

Udy, Stanley H. (1959): Organization of Work. A Comparative Analysis of Production among Nonindustrial Peoples, New Haven

Udy, Stanley H. (1965): The Comparative Analysis of Organizations, in: James G. March (Hrsg.), Handbook of Organizations, Chicago, S. 678-709

Udy, Stanley H. (1970): Work in Traditional and Modern Science, Englewood Cliffs

Watson, Rod (1997): Ethnomethodology and Textual Analysis, in: David Silverman (Hrsg.), Qualitative Research. Theory, Method and Practice, London et al., S. 80-88

Weber, Max (1968): Economy and Society, New York

Weber, Max (1976): Wirtschaft und Gesellschaft. Grundriß der verstehenden Soziologie, 5. rev. Auflage, Tübingen

Weiß, Christa (1987): Evaluating social problems: What have we learned?, in: Society, 25, 1, S. $40-45$

Wilensky, Harold (1956): Intellectuals in Labor Unions, Glencoe

Wimmer, Rudolf (1993): Zur Eigendynamik komplexer Organisationen. Sind Unternehmen mit hoher Eigenkomplexität steuerbar?, in: Gerhard Fatzer (Hrsg.), Organisationsentwicklung für die Zukunft. Ein Handbuch, Köln, S. 255-308

Wolcott, Harry F. (1995): The Art of Fieldwork, London

Wolff, Stephan (2000): Dokumenten- und Aktenanalyse, in: Uwe Flick/Ernst von Kardorff/Ines Steinke (Hrsg.), Qualitative Forschung. Ein Handbuch, Reinbek bei Hamburg, S. 502-513 